\title{
COVID-19 Detection using Deep Learning and Ultrasound Imaging
}

\author{
J.P. Gaidhani \\ Asstt. Prof., Department of \\ Information Technology \\ D.V.V.P.C.O.E. Ahmednagar, \\ Maharashtra
}

\author{
Harshada Gunjal \\ Department of Information \\ Technology \\ D.V.V.P.C.O.E. Ahmednagar, \\ Maharashtra
}

\author{
Sayli Waghmare \\ Department of Information \\ Technology \\ D.V.V.P.C.O.E. Ahmednagar, \\ Maharashtra
}

\author{
Akanksha Gatkal \\ Department of Information Technology, \\ D.V.V.P.C.O.E. Ahmednagar, Maharashtra
}

\author{
Gauri Kabra \\ Department of Information Technology, \\ D.V.V.P.C.O.E. Ahmednagar, Maharashtra
}

\begin{abstract}
There has been a rapid increase in growth of Covid-19 which has left a problem of efficient diagnosis and cheap diagnosis of Covid-19 patients. Using medical imaging techniques Like $\mathrm{CT}$ and X-ray combined with Deep learning are proving to be quite effective in the Diagnostic process. CT scans are widely used for the diagnostics they have been proven to be fast and have shown promising results and are sensitive even when the PCR test fails. But there are some flaws with CT scans like they are hard to sterilize, expensive and they are highly radiating. In this paper we have used ultrasound imaging technique which is cheaper, easy to use, fast and safe. We have Gathered Data set from various Sources of around 1000 images which consist of healthy lungs, Covid affected lungs and bacterial Pneumonia Affected Lungs. This has been assembled from various data sources which have been processed for deep learning Models and are open access. We have trained a deep learning model which hasa accuracy.
\end{abstract}

\section{Keywords}

Deep neural network, deep learning, COVID-19, CNN, PCR, CT-Scan, Patient Mobilization

\section{INTRODUCTION}

Till now, millions of people have become diagnosed by the corona virus epidemic and mortality is rising every day. As corona virus is extremely infective, it spreads rapidly among those linked to COVID-19 (Corona Virus Disease - 19) infected persons. The virus spills over saliva droplets or swab discharge from the nose while an affected individual with COVID-19 is tinging or sneezing. A COVID-19 afflicted individual may feel dry cough, muscle pain, headache, fever, sore throat, and gentle to moderate respiratory disease. Nevertheless, elderly adults and those with existing medical problems such as coronary disorder,asthma, chronic pulmonary disease, and cancer are most prone to experience severe illnesses.

Being an uncertain source of infection of the pneumonia form and a new virus to be created by mutation, it is very difficult for COVID-19 patients to find a remedy by vaccination or treatment. Further studies and social disparities within the community in high alerted regions in various countries impacted by the corona pandemic are also suggested as per the WHO. The origin of the 2019 corona virus outbreak is a novel corona virus, SARS-CoV-2, (COVID-19). Patient assessors may wish to order X-ray images to screen COVID19 findings, assess their severity, or to suggest additional disease etiologies. It will change the interest of the COVID-19 treaters through radiographic tests and may contribute to medical treatment or supportive therapeutic decisions, such as hospitalization, a requirement for additional supervision or expectation of disease risks. The American College of Radiography (ACR) recommends that

(i) The test for or first line treatment of COVID-19 ill not be performed using computed tomography (CT),

(ii) For admitted symptomatic patients with particular clinical signs of $\mathrm{CT}$, the $\mathrm{CT}$ will be used in a vigilant and reserved way,

(iii) Adequate protocols can be used for infectious management before future patients are screened,

(iv) Regular chest CT does not indicate that a person has no COVID-19 infection and pathological COVID 19 evaluation may not be accurate.

When used in the assessment of COVID 19 patients with symptoms of pneumonia in comparison to chest $\mathrm{x}$-rays, lung ultrasound (LUS) could provide increased diagnostic accuracy. LUS is particularly sensitive and sometimes passes chest x-rays of many pulmonary infections. The detection system for pneumonia and associated lung diseases has already been developed in lung ultrasound. The technique of diagnosing lung disease has been proposed as favored, particularly in resource-limited environments, such as crisesor low-income countries and has begun to substitute $\mathrm{x}$-rays for first-line testing. Since the outbreak ofCOVID-19, many researchers are trying to develop systems to detect the virus using radiographic images(CT scan or X-ray images). Very few researchers are involved in developing such systems using LUS images, cough sound, or Electrooculogram images. A multi-source system regarding COVID-19 detection has not been developed yet because all the available related datasets are singlesource. For example, there isno dataset where the CT scan images and the X-ray images are available from the same patient. As a datasetis a prerequisite to developing a system, currently, a multi-source system to combat COVID19 is verydifficult to realize. Instead, a system with different levels or layers of fusion can be a realistic one. Thereare some recent heterogeneous systems that deploy mass surveillance systems involving social distancingmeasures and body temperature measurements in a crowded place.Most of the COVID-19 screening systems are developed using deep learning, especially convolutionalneural networks (CNN) for 
images. Some systems used pre-trained CNN models, while some systems usedCNN models from scratch. There are two types of pre-trained $\mathrm{CNN}$ models:

(i) very deep and narrow, and

(ii) moderately deep and wide.

In the first type, the models consider that depth is the most important factor to increase accuracy. Someexamples are AlexNet and VGG Net. In the second type, the models consider that the width of the convolutional filters is the most important factor. Some examples of the second type are wide residual network and ResXNet. Both types have their advantages and disadvantages in terms of accuracy and number of parameters. To compromise between these two terms, a new term called the information density is sometimes used. The information density is calculated as a ratio between the accuracy and the number of parameters (in million) of a model. The recently proposed tree based deep model has a very high information density. One of the problems of a multi-stream CNN model is the increased computational complexity of the model. If we have three streams, the number of parameters is increased by approximately three times. Therefore, if there is a problem with the availability of a powerful computer, the multi-stream CNN model is often avoided. An alternative solution is to fuse features from different layers of a single CNN model. In this paper, a multilayer fusion approach of a CNN model is proposed for a COVID 19 screening system using LUS images. First, a light CNN model is proposed; the number of learnable parameters of the model is less than 0.4 million. Then features from different layers of the model are fused to produce a feature vector to be fed into a multilayer perception (MLP), in the form of a fully connected layer, for the classification. Various layers of fusion are also investigated. The major contributions of the paper are as follows:

(i) an efficient light CNN model is proposed for the COVID19 screening system,

(ii) a multi-layer features fusion approach is introduced in the system, and

(iii) an excellent information density is achieved by the system. It can be noted that the multi-layer features fusion is not common in any recently developed COVID-19 screening systems. Also, such systems using LUS images are rare in the literature.

\section{PROBLEM DESCRIPTION}

To use ultrasound images to detect and diagnose COVID-19 using deep learning and CNN technique. The dataset is divided into three lung classification which consist of ultrasound Imaging, pneumonia and healthy lungs.

In a pandemic situation such as COVID-19, due to a very large number of affected people, the screening system should be operated in less time, if not in real-time. The fusion of deep learned features is a very effective way to enhance the accuracy of a health related system. Typically, the fusion can be at three levels: feature level, classification level, and decision level. In the feature level fusion, a multi-stream CNN can be used and the features from all the streams are concatenated to produce a unified feature vector. The feature vector is then fed into a single classifier. In the classification level fusion, features from each stream are fed to a different classifier. The classifiers' outputs are then fused. In the decision level fusion, each classifier produces a decision, and then a majority voting type technique can be used to make the final decision based on the decisions of the classifiers.

\section{Objectives}

- The proposed research detects and diagnose of COVID19 using deep learning and $\mathrm{CNN}$ technique.

- To implement an automatic detection system as a quick alternative diagnosis option to prevent COVID-19. spreading among people and detection of corona virus pneumonia infected patient using Ultrasoundimages by designing a CNN model.

- The performance analysis of system which provides better prediction accuracy than other recommendationsystems.

\section{RELATED WORK}

Steenkiste et al. [9] developed a robust model focused on sensor fusion methods to enhance sleep apnea prediction. The system was used to gather and integrate reverse shortcut connections from ulti-sensor data including oxygen saturation, cardiac rhythm, thoracic breathing, and respiratory bands in the abdomen. The robustness and efficiency were tested in both CNN and LSTM prototypes.

Yang et al. [10] proposed a cross subject emotion recognition system using multi-sensors input. The sensors' data are vital signs such as body temperature and plethysmography, electroencephalogram (EEG)signals, electromyography (EMG) signals, and electrooculogram (EOG) signals. 10 different types of feature vectors were formed from these signals to produce a high dimensional feature space to be fed intoa support vector machine (SVM) classifier. Before feeding the features, a significant test was performed per feature to select the significant features only. Then, only the selected features were inputs to the SVM. Accuracies ranged between $72 \%$ and $89 \%$ were obtained using different datasets. Miao et al. [11] developed a cuffless blood pressure (BP) prediction system using double channel High pulse waves and a single channel electrocardiogram (ECG). 24 features from the pulse waves and 11 features from the ECG signal were extracted. Then a weakly supervised feature selection method selected the significant features, which were fed to a multi instance regression classifier to predict the BP. A private dataset of 85 patients having hypertension or hypotension was used to verify the system. Experimental results showed that the developed system achieved reliable prediction accuracy.

Guet al. [12] provided a design for the situation awareness recognition system to ensure the precision of the signal transmitting to a mine and the safety of mine employees. In the system, multi sensor data were analyzed and knowledge entropy theory was used to give various weights to different parameters of the data. A random forest (RF)SVM based classifier was used to predict the situation of the mine. Muzammalet. al. [13] provided coherent activity data that could be used for therapeutic research by introducing amultisensor data fusion system. The data collected from the body sensor networks (BSNs) were combined and incorporated into an ensemble classifier, which was installed in cloud computing. The evidenced based analysis found that a novel kernel random forest tree was the best performer in the system.

In [14], an audiovisual emotion recognition system was developed using $\mathrm{CNNs}$ and sparse auto encoder(SAE). Melcepstrum features with their temporal derivatives were extracted from audio signals and fed into a 2D CNN. Video signals were fed into a $3 \mathrm{D} \mathrm{CNN}$. The $\mathrm{CNN}$ features were fused using the SAE, followed by an SVM classifier. The system achieved good accuracy in various publicly available emotion datasets. 
Lin et al. [15] developed a hybrid multisensor fusion BSN framework to enable smart healthcare. The goal was to address the lack of conventional multi sensor fusion approaches in clinical uses. This research discussed in depth the basic roles of different layers of the framework. The first layer was a data preprocessing layer, where data were preprocessed using data cleaning, data conversion, data integration, and data compaction. In the fusion layer, an explainable neural network and Albased technique were usedto fuse data from cross domain and multi-sensor.

Amin et al. [16] proposed an EEG based motor imagery classification system by fusing different layers of a CNN model. In the system, the features from different layers were concatenated before being fed into a fully connected layer. The authors proved that fusing features from various layers achieved better results than without fusing. A system of medical image fusion relying on a Rolling Guidance Filtering (RGF) was introduced in[17]. The system used the RGF to transform the source artifacts into either geometric or detailed elements. The types of fusion were used. A linear prediction based fusion was used for the geometric component, while a sum-modified Laplacian was used for the system had its own fusion role; the LP-based fusion rule was used for the structural components and the Sum-Modified-Laplacian was used for the detailed components. The system had good accuracy compared with other relevant systems.

Simjanoska et al. [18] proposed a multi-level fusion system to predict BP from the ECG sensor data. In this system, the process was summarized into five separate levels. In level 1, data were combined from various ECG sensors, and in level 2, data generated features were derived using separate approaches. The combining of feedback knowledge from seven separate classifiers was included in level 3. With each BP type, knowledge from multi-target regression models was integrated into level 4 , and then a single prediction was obtained in level 5 using the probability outputs of the models.

The challenge of obtaining structural and functional knowledge from both MRI and PET images by the usage of the same decomposition technique is present in multi-scale transform fusion approaches. To address the challenge, Du et al. [19] introduced a method called the intrinsic image decomposition. The method decomposed MRI and PET images into two-scales in the spatial domain. Then the original image coefficients were integrated with these twoscales elements. Experiments were performed on both gray and pseudo-color imagesand the obtained accuracies were good.

LUS images are typically low-resolution monochrome images. Roy et al. proposed a network using deep space transformer, which simultaneously forecasts the magnitude of the disease correlated with the input LUS images and provide a weakly regulated localization of pathological artifacts for COVID-19 screening. Besides, the authors proposed a way of aggregating frame-level scores to predict video-level ultrasound inputs. The network gave $60 \%$ precision and $70 \%$ recall in the case of video-level inputs.

Born et al. recently developed a deep CNN named POCOVID-Net to screen COVID-19 using LUS images [24]. The authors first developed an annotated LUS images dataset by collecting images from different sources. The named the dataset as the POCUS (point of care ultrasound) dataset. The dataset is available online to use publicly. POCOVID-Net used an extension of VGG-16 Net and achieved $86 \%$ precision and $78 \%$ recall on the dataset.

Cristina et al. developed a CNN model to quantify the assessment of B-lines in LUS images [25]. B-lines corresponding to alveolar interstitial syndrome may appear in COVID-19 or pneumonit is diseases. The model consisted of eight intermediate layers having 3D filters followed by fullyconnected layers. Severity measurement was also performed using the model.A CNN model to classify five major lung features linked to abnormal lung conditions: B-lines, converged B-lines, lack of pulmonary sliding, consolidation, and pleural effusion was proposed in . The model was trained with closely measured lung conditions of pig's LUS videos. Pneumothorax (absence of pulmonary sliding) was observed with the CNN Inception V3 utilizing virtual M-mode images. A single shot detection (SSD) system had been used to monitor the other features. A computational method was developed for COVID-19 diagnosis system using CNNs. Throughout the secure management of COVID-19spreads, the usage of ultrasound is now important as it can facilitate the clinical assessment and lung imagingto be conducted simultaneously at a single doctor's bedside. Several other systems based on deep learning using LUS images have shown promising results in different applications such as the detection of lung cancer, thyroid papillary cancer, and pneumothorax detection.

\section{SYSTEM ARCHITECTURE}

The Covid 19 in a year has affected hundreds of millions of people worldwide and has caused a chaos all around the world and has caused stress to people and all the medical professionals. The best cure found until now is only the fast diagnostic techniques that can help to stop the spread. Biomedical imaging techniques have great potential in tackling the convention methods of Covid testing such as RTPCR and IGM/G specifically. There are studies that show that $\mathrm{CT}$ scan can detect Covid at higher sensitivity rates than RT-PCR. While CT scan is the best tool in which X-rays are still used as first line of diagnostics. The Lungs Ultrasound Imaging is already an Established method for monitoring Pneumonia and other lung diseases. The strengths of ultrasound are enormous like its simplicity in execution, the ease of repeatability and its execution with reallocation. The Diagnosis can be fastened by connecting the system to the cloud and uploading the images instantly to the cloud. There was lot of work done for automatic detection of Covid using XRAY and CT-scan but less work has been done using Ultra sound images. The proposed system consists of CNN to tackle the task of Covid detection using ultrasound. Uses VGG16as the base for the model; this deep neural network has been demonstrated to be useful in various deep learning neural networks. Then it is followed by a layer of hidden layer of 64 neurons of Rely activation, which has a drop out of 0.5 and batch normalization, and a final output layer of softmax. Then the model is trained on the Image net which has extracted features like shapes and textures. All images are resizes in 224_224 and fed through the network. Then the model is trained with cross entropy. The data augmentation techniques are used to change and diversify the dataset. Image Data Generator is also used which does a random transformation on the data set images while generating a batch. There are following transformations used to allow rotation of 10 degree of image height and weight. Augmentation is diverse and valuable to prevent over fitting. The model is trained to differentiate between healthy, Covid19 and Pneumonia patients. The pre-trained dataset used by VGG 16 where only defined last 64 neuron for training purposes. 


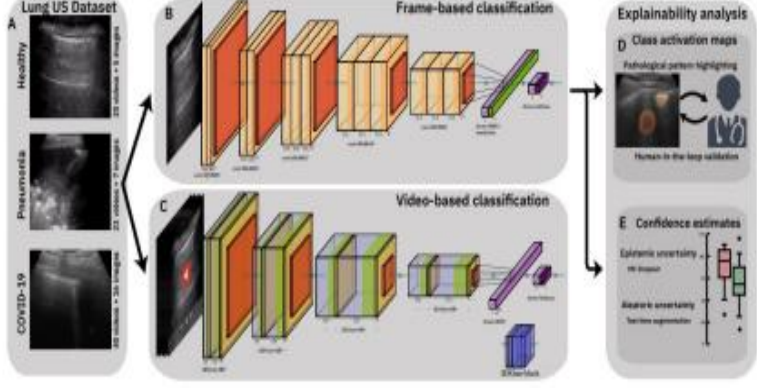

Fig1.System Architecture

\subsection{Dataset Used}

The preprocessed dataset is collected which consist of 64 lungs ultrasound recording divided into 11, 14and 39 of healthy patient, pneumonia and Covid 19 respectively where $60 \%$ data is COVID 19 images. The linear probes give high resolution images. In Proposed system only the convex probes data are used because the data set available majorly consist of convex probes images. For deep organ convex probes areused more. Ideally for lungs linear probes should be used but more hospitals are equipped with convexprobes.Ultrasound imaging has widely been used in diagnosis in the field of cardiology and recently covered many other organs. The Ultrasound has been popularized due to advancements in technical space like machine learning algorithms instead of its low quality and high signal to noise ratio. Many researches have used ultrasound for detecting different types of cancers like Pancreatic, lungs, breast and prostate. Using Computer Aided Diagnosis for Pulmonary Pathologies is a field of research using X-ray images that has in existence since the 1960. Although X Ray Imaging is widely used for detection of many lung diseases and it is not suitable for Covid detection in earlier stages. Instead it has useful for bilateral multi focal consolidation and partially fused into massive consolidation.

Table 4.1: Sources of Dataset

\begin{tabular}{|l|l|l|}
\hline $\begin{array}{l}\text { Data } \\
\text { Source }\end{array}$ & Data Selection & Website Description \\
\hline Grepmed & $\begin{array}{l}\text { 10 COVID-19,9 } \\
\text { pneumonia and 2 } \\
\text { healthyvideos }\end{array}$ & $\begin{array}{l}\text { GrepMed is ablemedical image } \\
\text { communitysourced, search } \\
\text { ablemeding } \\
\text { repository for referencing } \\
\text { clinically } \\
\text { relevant medical images }\end{array}$ \\
\hline $\begin{array}{l}\text { ThePocus } \\
\text { Atlas }\end{array}$ & $\begin{array}{l}\text { 8 COVID-19,3 } \\
\text { pneumoniaand 3 } \\
\text { healthy videos }\end{array}$ & $\begin{array}{l}\text { The PocusAtlas is a } \\
\text { Collaborative Ultra sound } \\
\text { Education } \\
\text { Platform }\end{array}$ \\
\hline
\end{tabular}

\section{CONCLUSION}

It is believed that it has helped radiologists to make decisions in clinical practice due to the higherperformance in order to detect COVID-19 at an early stage so we proposed a deep learning based approachusing LUS images. LUS performed by the physician gives a safe, inexpensive, easily reproducible technique with a great potential in allowing to differentiate patients with signs of COVID-19 orpneumonia. Our contribution is the exploration of automatizing COVID-19 detection from lung ultrasoundimaging in order to provide a quick assessment of the possibility of a person to be infected
withCOVID-19. The developed model was effective in extracting features from an $\mathrm{x}$-ray image and forecast the occurrence or non-existence of COVID-19.

\section{REFERENCES}

[1] WHO, "Laboratory testing strategy recommendations for COVID19: Interim guidance," Tech. Rep.,2020. [Online]. Available: https://apps.who.int/iris/bitstream/handle/10665/331509/ WHOCOVID-19-lab testing-2020.1-eng.pdf

[2] Deepak A Vidhate, Parag Kulkarni, "Performance comparison of multiagent cooperative reinforcement learning algorithms for dynamic decision making in retail shop application", International Journal of Computational Systems Engineering, Inderscience Publishers (IEL), Volume 5, Issue 3, pp 169-178, 2019.

[3] R. Niehus, P. M. D. Salazar, A. Taylor, and M. Lipsitch, "Quantifying bias of COVID-19 prevalence and severity estimates in Wuhan, China that depend on reported cases in international travelers," medRxiv, p.2020.02.13, feb 2020.

[4] Deepak A Vidhate, Parag Kulkarni, "A Framework for Dynamic Decision Making by Multi-agent Cooperative Fault Pair Algorithm (MCFPA) in Retail Shop Application", Information and Communication Technology for Intelligent Systems, Springer, Singapore, pp 693-703, 2019.

[5] Y. Yang et al., "Evaluating the accuracy of di_erent respiratory specimens in the laboratory diagnosis and monitoring the viral shedding of $2019-\mathrm{nCoV}$ infections," medRxiv, p. 2020.02.11., feb 2020

[6] Deepak A Vidhate, Parag Kulkarni, "A Novel Approach by Cooperative Multiagent Fault Pair Learning (CMFPL)" Communications in Computer and Information Science, Springer, Singapore, Volume 905, pp 352-361,2018.

[7] M. E. H. Chowdhury et al., "Can AI Help in Screening Viral and COVID-19 Pneumonia?" in IEEE Access, vol. 8, pp. 132665-132676, 2020, DOI: 10.1109/ACCESS.2020.3010287.

[8] Deepak A. Vidhate and Parag Kulkarni, "Expertise Based Cooperative Reinforcement Learning Methods (ECRLM)", International Conference on Information \& Communication Technology for Intelligent System, Springer book series Smart Innovation, Systems and Technologies(SIST, volume 84), Cham, pp 350-360, 2017.

[9] J. C. Monteral. (2020). COVID-Chestxray Database. Available: https://github.com/ieee8023/covid-chestxraydataset

[10] Deepak A. Vidhate and Parag Kulkarni "Innovative Approach Towards Cooperation Models for Multi-agent Reinforcement Learning (CMMARL) "International Conference on Smart Trends for Information Technology and Computer Communications Springer, Singapore, pp. 468-478, 2016.

[11] Alqudah, Ali Mohammad; Qazan, Shoroq (2020), "Augmented COVID-19 X-ray Images Dataset”,Mendeley Data, v4 http://dx.doi.org/10.17632/2fxz4px6d8.4 
[12] Deepak A. Vidhate and Parag Kulkarni, "Enhanced Cooperative Multi-agent Learning Algorithms (ECMLA) using Reinforcement Learning" International Conference on Computing, Analytics and Security Trends (CAST), IEEE Xplorer, pp 556 - 561, 2017.

[13] Abiyev R. H., Ma'aitah M. K. S., "Deep Convolutional Neural Networks for Chest Diseases Detection",Journal of Healthcare Engineering, 2018.

[14] Deepak A. Vidhate and Parag Kulkarni, "Exploring Cooperative Multi-agent Reinforcement Learning Algorithm (CMRLA) for Intelligent Traffic Signal Control", Smart Trends in Information Technology and Computer Communications. SmartCom 2017, Volume 876, pp 71-81,2018.

[15] T. Liang, "Handbook of COVID-19 prevention and treatment," The First Affiliated Hospital, ZhejiangUniversity School of Medicine Compiled According to Clinical Experience, 2020.

[16] Deepak A Vidhate and Parag Kulkarni, "A Novel Approach for Dynamic Decision Making by Reinforcement Learning-Based Cooperation Methods (RLCM)", International Conference on Intelligent Computing and Applications, Springer, Singapore, 401$411,2018$.

[17] Soyoun Kim, Dong-Min Kim, and Baeckseung Lee. Insufficient sensitivity of rna dependent rna polymerase gene of sars-cov-2 viral genome as confirmatory test using korean covid-19 cases. 2020.

[18] Deepak A Vidhate and Shruti S Pophale, "Depression Scale Recognition Over Fusion of Visual and Vocal
Expression using Artificial Intellectual Method", International Journal of Computer Applications (IJCA), Volume 183, Issue 24, Pages 16-19,2021.

[19] Roman Kalkreuth and Paul Kaufmann. Covid-19: A survey on public medical imaging data resources.arXiv preprint arXiv:2004.04569, 2020.

[20] Deepak A Vidhate and Mansi Palve, "Customer Relationship Management: An IT Success as Multifunctional Domain and it's Fututre Directions", International Journal of Computer Applications (IJCA), Volume 183, Issue 19, Pages 30-34,2021.

[21] MJ Smith, SA Hayward, SM Innes, and A Miller. Pointof-care lung ultrasound in patients with covid19-a narrative review. Anaesthesia, 2020

[22] MdZahangirAlom, MM Shaifur Rahman, MstShamimaNasrin, Tarek M Taha, and Vijayan K Asari.Covidmtnet : Covid19detectionwithmultitaskdeeplearningapproaches:arXiv; pagesarXiv 2004 2020:

[23] Jianpeng Zhang, YutongXie, Yi Li, Chunhua Shen, and Yong Xia. Covid-19 screening on chest $\mathrm{x}$-ray images using deep learning based anomaly detection. arXiv preprint arXiv:2003.12338, 2020.

[24] Paulo ViniciusMasnik, Roberto Alexandre Dias and Mario De Noronha Neto. Detection of Myocardial Infarction in Electrocardiograms using Machine Learning. International Journal of Computer Applications 183(9):12-19, June 2021. 